\title{
Certification of automated termination proofs *
}

\author{
Evelyne Contejean ${ }^{1}$, Pierre Courtieu ${ }^{2}$, Julien Forest ${ }^{2}$, Olivier Pons ${ }^{2}$, and \\ Xavier Urbain ${ }^{2}$ \\ 1 LRI, Université Paris-Sud, CNRS, INRIA Futurs, Orsay F-91405 \\ ${ }^{2}$ CÉDRIC - Conservatoire national des arts et métiers
}

\begin{abstract}
Nowadays, formal methods rely on tools of different kinds: proof assistants with which the user interacts to discover a proof step by step; and fully automated tools which make use of (intricate) decision procedures. But while some proof assistants can check the soundness of a proof, they lack automation. Regarding automated tools, one still has to be satisfied with their answers Yes/No/Do not know, the validity of which can be subject to question, in particular because of the increasing size and complexity of these tools.

In the context of rewriting techniques, we aim at bridging the gap between proof assistants that yield formal guarantees of reliability and highly automated tools one has to trust. We present an approach making use of both shallow and deep embeddings. We illustrate this approach with a prototype based on the CiME rewriting toolbox, which can discover involved termination proofs that can be certified by the COQ proof assistant, using the COCCINELLE library for rewriting.
\end{abstract}

\section{Introduction}

Formal methods play an increasingly important role when it comes to guaranteeing good properties for complex, sensitive or critical systems. In the context of proving, they rely on tools of different kinds: proof assistants with which the user interacts step by step, and fully automated tools which make use of (intricate) decision procedures.

Reducing the cost of formal proofs amounts to using more and more automation. However, while some proof assistants can check the soundness of a proof, one still has to be satisfied with the answer of automated tools. Yet, since application fields include possibly critical sectors as security, code verification, cryptographic protocols, etc., reliance on verification tools is crucial.

Some proof assistants, like COQ [29], need to check mechanically the proof of each notion used. Among the strengths of these assistants are firstly a powerful specification language that can express both logical assertions and programs, hence properties of programs, and secondly a highly reliable procedure that checks the soundness of proofs.

For instance, COQ or ISABELLE/HOL [27] have a small and highly reliable kernel. In COQ, the kernel type-checks a proof term to ensure the soundness of a proof. Certified-programming environments based on these proof assistants find here an additional guarantee. Yet, among the weaknesses of these assistants, one may regret the lack of automation in the proof discovery process. Automation is indeed difficult to obtain

\footnotetext{
* Work partially supported by A3PAT project of the French ANR (ANR-05-BLAN-0146-01).
} 
in this framework: the proof assistant has to check a property proven by an external procedure before accepting it. Therefore, such a procedure has to return a proof trace checkable by the assistant.

We want to meet the important need of proofs delegation for some properties in the framework of rewriting techniques. We will focus on generic ways to provide reasonably-sized proof traces for complex properties, for instance termination.

Termination is the property of a program any execution of which always yields a result. Fundamental when recursion and induction are involved, it is an unavoidable preliminary for proving many various properties of a program. Confluence of a rewriting system, for instance, becomes decidable when the system terminates. More generally, proving termination is a boundary between total and partial correctness of programs. Hence, automating termination is of great interest for provers like CoQ, in which functions can be defined only if they are proven to be terminating.

The last decade has been very fertile w.r.t. automation of termination proofs, and yielded many efficient tools (APROVE [17], CiME [8], JAMBOX [15], TPA [22], TTT [20] and others) referenced on the website of the Termination Competition [25]. Some of them display nice output for human reading. However, there is still a clear gap between proof assistants that provide formal guarantees of reliability and highly automated tools that do not. In the sequel, we aim at bridging this gap.

We present here a methodology for the particularly important challenge of automatically generating proof traces in the domain of first order term rewrite systems and in particular for termination proofs of such systems. We do not restrict to classical approaches of all-shallow embedding or, like Color [4], of all-deep embedding to model properties or techniques. Instead we use a mixed approach so as to get the best of both worlds. We implemented our principles and methodology within the version 2.99 of the $\mathrm{CiME}$ rewrite tool box. This version uses parts of the termination engine of CiME2.04, with our mixed approach it can certify (with COQ) termination proofs of more than 350 problems of the TPDB, using involved criteria. This is made possible thanks to a COQ library for rewriting (COCCINELLE) developed by E. Contejean in our project.

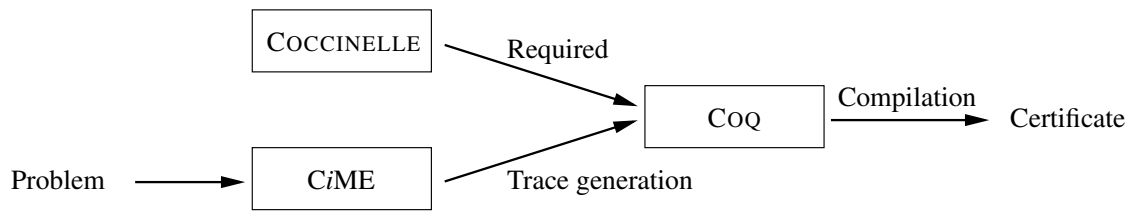

We make our notations precise and give some prerequisites about first order term rewriting and about the CoQ proof assistant in Section 2. Then, in Section 3, we present our modelling of termination of rewriting in COQ, which mixes deep and shallow embeddings in order to take benefits of both. We briefly present the COQ library COCCINELLE developed in the project to that purpose. In section 4 we present the certification of proofs using involved criteria such as Dependency Pairs [1] with graphs refinement, mixing orderings based on polynomial interpretations [23] or RPO [10] with AFS [1]. We shall adopt the end-user point of view and provide some experimental results of CiME 2.99 in Section 5. Eventually we briefly compare with related works and conclude in Section 6. 


\section{Preliminaries}

\subsection{Rewriting}

We assume the reader familiar with basic concepts of term rewriting $[2,13]$ and termination, in particular with the Dependency Pairs (DP) approach [1]. We recall usual notions, and give notations. A signature $\mathcal{F}$ is a finite set of symbols with arities. Let $X$ be a countable set of variables; $T(\mathcal{F}, X)$ denotes the set of finite terms on $\mathcal{F}$ and $X$. $\Lambda(t)$ is the symbol at root position in term $t$. We write $\left.t\right|_{p}$ for subterm of $t$ at position $p$ and $t[u]_{p}$ for term $t$ where $\left.t\right|_{p}$ has been replaced by $u$. Substitutions are mappings from variables to terms and $t \sigma$ denotes the application of a substitution $\sigma$ to a term $t$.

A term rewriting system (TRS for short) over a signature $\mathcal{F}$ is a set $R$ of rewrite rules $l \rightarrow r$ with $l, r \in T(\mathcal{F}, X)$. A TRS $R$ defines a monotonic relation $\rightarrow_{R}$ closed under substitution (aka a rewrite relation) in the following way: $s \rightarrow_{R} t$ ( $s$ reduces to $t$ ) if there is a position $p$ such that $\left.s\right|_{p}=l \sigma$ and $t=s[r \sigma]_{p}$ for a rule $l \rightarrow r \in R$ and a substitution $\sigma$. In the following, we shall omit systems and positions that are clear from the context. We denote the reflexive-transitive closure of a relation $\rightarrow$ by $\rightarrow^{\star}$. Symbols occurring at root position in the left-hand sides of rules in $R$ are said to be defined, the others are said to be constructors.

A term is $R$-strongly normalizable ( $R$-SN) if it cannot reduce infinitely many times for the relation defined by System $R^{1}$. A rewrite relation terminates if any term is $\mathrm{SN}$. Termination is usually proven with the help of reduction orderings [11] or ordering pairs with dependency pairs. The set of unmarked dependency pairs ${ }^{2}$ of a TRS $R$, denoted $D P(R)$ is defined as $\left\{\langle u, v\rangle\right.$ such that $u \rightarrow t \in R$ and $\left.t\right|_{p}=$ $v$ and $\Lambda(v)$ is defined $\}$. An ordering pair is a pair $(\succeq,>)$ of relations over $T(\mathcal{F}, X)$ such that: 1) $\succeq$ is a quasi-ordering, i.e. reflexive and transitive, 2) $>$ is a strict ordering, i.e. irreflexive and transitive, and 3 ) $>\cdot \succeq=>$ or $\succeq \cdot>=>$. An ordering pair is well-founded if there is no infinite strictly decreasing sequence $t_{1}>t_{2}>\ldots$

\subsection{The COQ proof assistant}

The CoQ proof assistant is based on type theory and features: 1) A formal language to express objects, properties and proofs in a unified way; all these are represented as terms of an expressive $\lambda$-calculus: the Calculus of Inductive Constructions (CIC) [9]. $\lambda$-abstraction is denoted fun $\mathrm{x}: \mathrm{T} \Rightarrow \mathrm{t}$, and application is denoted $t$ u. 2) A proof checker which checks the validity of proofs written as CIC-terms. Indeed, in this framework, a term is a proof of its type, and checking a proof consists in typing a term. The tool's correctness relies on this type checker, which is a small kernel of 5000 lines of OBJECTIVE CAML code.

For example the following simple terms are proofs of the following (tautological) types (remember that implication arrow $\rightarrow$ is right associative): the identity function fun $x: A \Rightarrow x$ is a proof of $A \rightarrow A$, and fun $(x: A)(f: A \rightarrow B) \Rightarrow f x$ is a proof of $\mathrm{A} \rightarrow(\mathrm{A} \rightarrow \mathrm{B}) \rightarrow \mathrm{B}$.

\footnotetext{
${ }^{1}$ When $R$ is clear from the context, we shall write $\mathrm{SN}$.

${ }^{2}$ For readability's sake we detail only unmarked DP, see Sec. 4.4 for how we deal with marks.
} 
A very powerful feature of $\mathrm{COQ}$ is the ability to define inductive types to express inductive data types and inductive properties. For example the following inductive types define the data type nat of natural numbers, $O$ and $S$ (successor) being the two constructors $^{3}$, and the property even of being an even natural number.

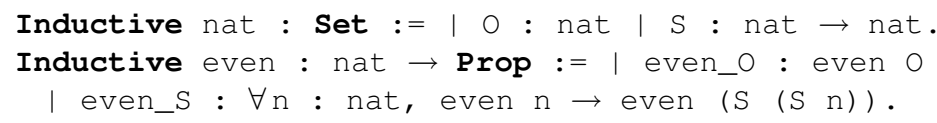

Hence the term even_S ( $S(S O))$ (even_S O (even_O)) is of type even $(S(S(S(S O))))$ so it is a proof that 4 is even.

\subsection{Termination in CoQ}

We focus in this paper on termination. This property is defined in COQ standard library as the well-foundedness of an ordering. Hence we model TRS as orderings in the following. This notion is defined using the accessibility predicate. A term $t: A$ is accessible for an ordering $<$ if all its predecessors are, and $<$ is well-founded if all terms of type $A$ are accessible ( $\mathrm{R}$ y $\mathrm{x}$ stands for $y<x$ ):

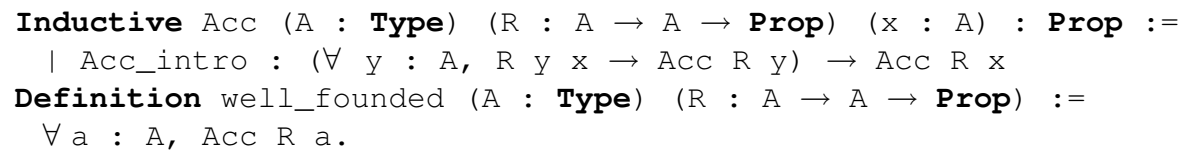

\section{This inductive definition contains both the basis case (that is when an element has no predecessor w.r.t. the relation $R$ ) and the general in- ductive case. For example, in a relation $\mathrm{R}$ on bool defined by} $R$ true false, true is accessible because it has no predecessor, and so is false because its only predecessor is true. Hence ACC R true and AcC R false are provable, hence well-founded $R$ is provable. The usual ordering $<$ is well-founded over the natural numbers, since 0 has no predecessor, and by structural induction, if $n$ is accessible, so is $S(n)$; whereas < is not well-founded over the integers.

\section{Modelling termination of rewriting in $\mathrm{COQ}$}

If $\mathrm{R}$ is the relation modelling a TRS $\mathcal{R}$, we should write $\mathrm{R} u t$ (which means $u<t$ ) when a term $t$ rewrites to a term $u$. For the sake of readability we will use as much as possible the CoQ notation: $t-[\mathrm{R}]>u\left(\right.$ and $t-[\mathrm{R}] *>u$ for $t \rightarrow^{*} u$ ) instead.

The wanted final theorem stating that $\mathcal{R}$ is terminating has the following form:

\footnotetext{
${ }^{3}$ Note that this notion of constructors is different from the one in Section 2.1.
} 
Theorem well_founded_R: Well_founded R.

Since we want certified automated proofs, the definition of $R$ and the proof of this theorem are discovered and generated in COQ syntax with full automation by our prototype. In order to ensure that the original rewriting system $\mathcal{R}$ terminates, the only things the user has to check is firstly that the generated relation $\mathrm{R}$ corresponds to $\mathcal{R}$ (which is easy as we shall see in Section 3.2), and secondly that the generated COQ files do compile.

\subsection{Shallow vs deep embedding}

In order to prove properties on our objects (terms, rewriting systems, polynomial interpretations...), we have to model these objects in the proof assistant by defining a theory of rewriting. There are classically two opposite ways of doing this: shallow embedding and deep embedding. When using shallow embedding, one defines ad hoc translations for the different rewriting notions, and proves criteria on the translation of each considered system. When using deep embedding, one defines generic notions for rewriting and proves generic criteria on them, and then instantiates notions and criteria on the considered system. Both shallow and deep embedding have advantages and drawbacks. On the plus side of shallow embedding are: an easy implementation of rewriting notions, and the absence of need of meta notions (as substitutions or term well-formedness w.r.t. a signature). On the minus side, one cannot certify a criterion but only its instantiation on a particular problem, which often leads to large scripts and proof terms. Regarding deep embedding, it usually leads (not always as we explain below) to simpler scripts and proof terms since one can reuse generic lemmas but at the cost of a rather technical first step consisting in defining the generic notions and proving generic lemmas.

We present here an hybrid approach where some notions are deep ( $\Sigma$-algebra, RPO) and others are shallow (rewriting system, dependency graphs, polynomial interpretations). The reason for this is mainly due to our proof concern which makes sometimes deep embedding not worth the efforts it requires: some premises of generic lemmas, which have to be proven on each considered problem, are as hard (if not harder) to prove than the shallow lemma itself. We will show that using both embeddings in a single proof is not a problem, and moreover that we can take full benefit of both.

\subsection{The Coccinelle library}

The deep part of the modelling is formalised in a public CoQ library called CocCINELLE [6]. To start with, it contains a modelling of the mathematical notions needed for rewriting, such as term algebras, generic rewriting, generic and AC equational theories and RPO with status. It contains also proofs of properties of these notions, for example that RPO is well-founded whenever the underlying precedence is so.

Moreover CoccinelLE is intended to be a mirror of the CiME tool in CoQ; this means that some of the types of CocCINELLE (terms, etc.) are translated from CiME (in OBJective CAML) to CoQ, as well as some functions (AC matching) ${ }^{4}$. Trans-

\footnotetext{
${ }^{4}$ It should be noticed that COCCINELLE is not a full mirror of CiME: some parts of CiME are actually search algorithms for proving for instance equality of terms modulo a theory or termination of TRSs. These search algorithms are much more efficient when written in OBJECTIVE CAML than in COQ, they just need to provide a trace for COCCINELLE.
} 
lating functions and proving their full correctness obviously provide a certification of the underlying algorithm. Note that some proofs may require that all objects satisfying a certain property have been built: for instance in order to prove local confluence of a TRS, one need to get all critical pairs, hence a unification algorithm which is complete ${ }^{5}$.

Since module systems in OBJeCtive CAML and COQ are similar, both CiME and COCCINELle have the same structure, except that CiME contains only types and functions whereas COCCINELLE also contains properties over these types and functions.

Terms A signature is defined by a set of symbols with decidable equality, and a function arity mapping each symbol to its arity.

The arity is not simply an integer, it mentions also whether a symbol is free of arity $n, \mathrm{AC}$ or $\mathrm{C}$ (of implicit arity 2) since there is a special treatment in the $\mathrm{AC} / \mathrm{C}$ case.

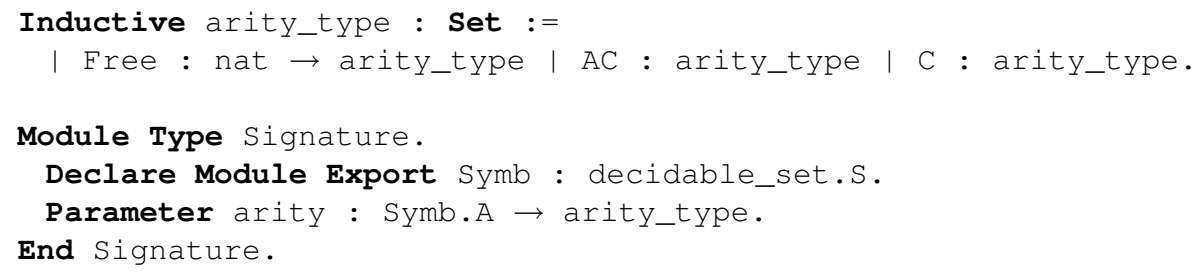

Up to now, our automatic proof generator does not deal with $\mathrm{AC}$ nor $\mathrm{C}$ symbols, hence in this work all symbols have an arity Free n. However, AC/C symbols are used in other parts of COCCINELLE, in particular the formalisation of AC matching [5].

A term algebra is a module defined from its signature $\mathrm{F}$ and the set of variables $\mathrm{X}$.

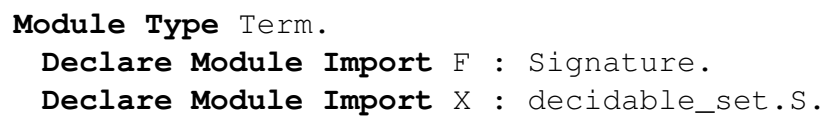

Terms are defined as variables or symbols applied to lists of terms. Lists are built from two constructors $\mathrm{nil}$ and : :, and enjoy the usual $[\mathrm{x} ; \mathrm{y} ; \ldots]$ notation.

Inductive term : Set :=

| Var : variable $\rightarrow$ term | Term : symbol $\rightarrow$ list term $\rightarrow$ term.

This type allows to share terms in a standard representation as well as in a canonical form; but this also implies that terms may be ill-formed w.r.t. the signature. The module contains decidable definitions of well-formedness. However, the rewriting systems we consider do not apply on ill-formed terms, so we will not have to worry about it to prove termination.

The term module type contains other useful definitions and properties that we omit here for the sake of clarity. The Coccinelle library contains also a functor term. Make which, given a signature and a set of variables, returns a module of type Term. We will not show its definition here.

Module Make (F1 : Signature) (X1 : decidable_set.S) : Term.

\footnotetext{
${ }^{5}$ Local confluence is not part of CocCINELLE yet.
} 
Rewriting systems TRSs provided as sets of rewrite rules are not modelled directly in COCCINELlE. Instead, as explained in the introduction of this Section, we use orderings built from any arbitrary relation $R$ : relation term (by definition relation $A$ is $\mathrm{A} \rightarrow \mathrm{A} \rightarrow$ Prop). The usual definition can be retrieved obviously from a list of rewrite rules (i.e. pairs of terms) $\mathcal{R}$ by defining $\mathrm{R}$ as:

$$
\forall s, t \in T(\mathcal{F}, X), s-[\mathrm{R}]>t \Longleftrightarrow(s \rightarrow t) \in \mathcal{R}
$$

The COCCINELLE library provides a module type RWR which defines a reduction relation (w.r.t. the "rules" R) and its properties.

Module Type RWR.

Declare Module Import $\mathrm{T}$ : Term.

The first step toward definition of the rewrite relation is the closure by instantiation:

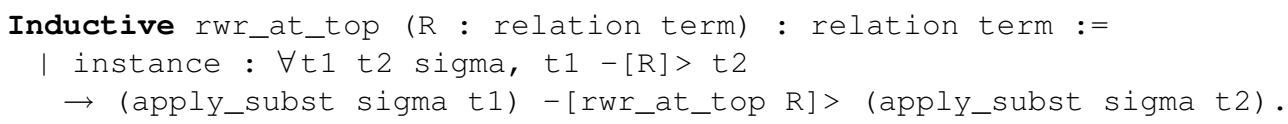

Then we define a rewrite step as the closure by context of the previous closure. Notice the use of mutual inductive relations to deal with lists of terms.

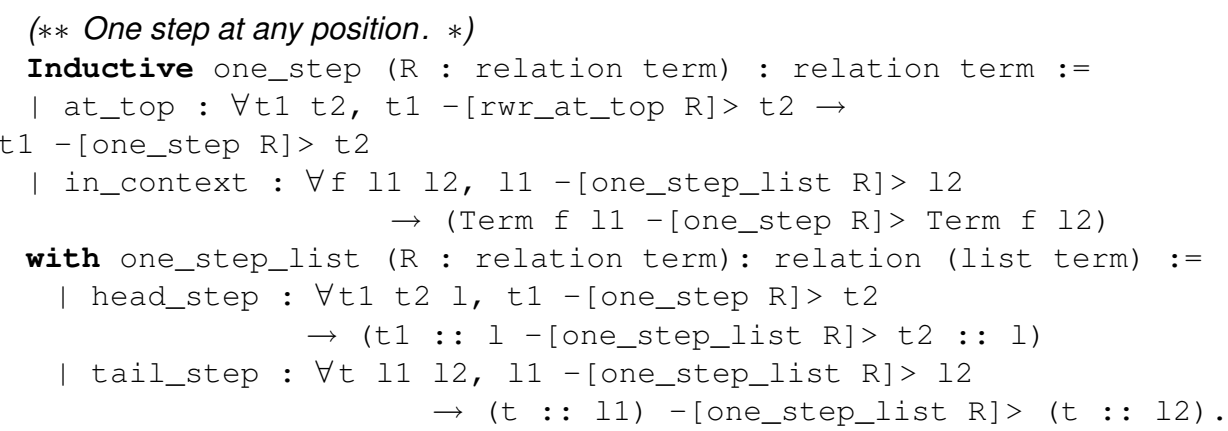

This module type contains properties declared using the keyword Parameter. This means that to build a module of this type, one must prove these properties. For instance it contains the following property stating that if $t_{1} \rightarrow^{+} t_{2}$ then $t_{1} \sigma \rightarrow^{+} t_{2} \sigma^{6}$ for any substitution $\sigma$.

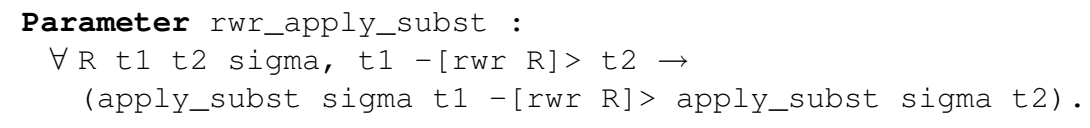

The library contains a functor rewriting. Make building a module of type RWR from a module $\mathrm{T}$ of type Term. This functor builds in particular the proof of all properties required by RWR. For an R representing the rules of the TRS under consideration, the final theorem we want to generate is:

Theorem Well_founded_R: Well_founded (one_step R).

To ensure that one_step R corresponds to the original TRS $\mathcal{R}$, it suffices for the user to perform the easy check that $\mathrm{R}$ corresponds to the set of rules defining $\mathcal{R}$.

\footnotetext{
${ }^{6}$ The transitive closure of one_step is defined as rwr in CocCINELLE.
} 
Note that since the datatype term represents any $\Sigma$-algebra (via application of the functor Make), we can say that terms are represented in a deep embedding. However, to simplify proofs, we avoid using substitutions by quantifying on subterms as much as possible. That makes our use of the type term slightly more shallow on this point.

\section{Generation of proof traces}

We will illustrate our approach by presenting proof generation techniques at work on a small example in our prototype, namely CiME 2.99. While being based on the CiME2 tool box, this prototype does not certify all its predecessor's termination power. For instance, modular criteria [30] and termination modulo equational theories are not supported yet. In the following, we restrict to (marked/unmarked) Dependency Pairs [1] with/without graphs refinements. The orderings we deal with include strictly the orderings that $\mathrm{C} i \mathrm{ME}$ generates: (non-linear) polynomial interpretations (section 4.6) and RPO with status ${ }^{7}$ (section 4.7).

\subsection{Global structure of a generated proof}

A close look at different termination tools reveals a common underlying methodology which we use as the skeleton of our generated proofs. It consists in deriving recursively from a relation $R$ a set of other relations $R_{i}$ such that if all $R_{i} s$ are terminating, then so is $R$. For instance, this structure appears explicitly with the processors [18] of APROVE.

This recursive decomposition is done using termination criteria like DP criteria, (complex) graph criteria, modular criteria, etc. Some tools may use some backtracking but if the procedure succeeds, it means that an implicit tree was built:

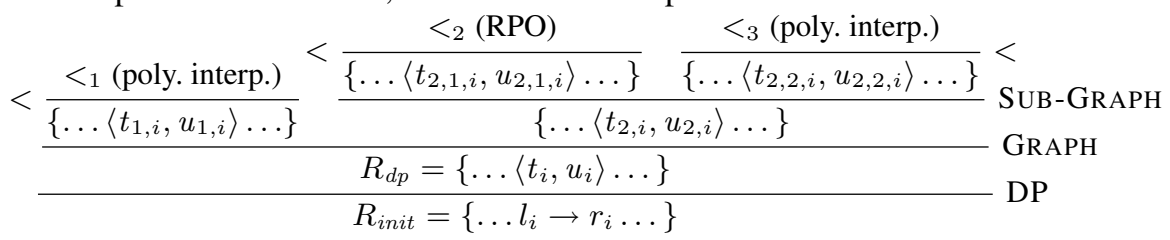

This tree is rooted by the initial problem, namely the initial rewriting system and the first termination criterion used. Each intermediate node is also labelled by a relation and the termination criterion used to decompose the node into its children. Finally each leaf must be labelled by a relation $R_{i}$ and a well-founded ordering which includes it.

The tree structure is reflected in the generated file. Indeed, for each criterion step ( $R$ replaced by sufficient conditions $\left\{R_{i}\right\}$ ), we will generate a lemma of the form:

Lemma wf_R_if_wf_Ri : well_founded R1 $\rightarrow$ well_founded R2 . . $\rightarrow$ well_founded $R$.

The proof of this lemma depends on the termination criterion used (Sec. 4.4 and 4.5).

Each time a leaf is proven using an ordering, we generate a lemma of the form:

Lemma wf_Ri : well_founded Ri.

\footnotetext{
${ }^{7}$ To date, CiME can discover polynomials and LPO with AFS.
} 
The proof is made by induction on the ordering built by the automated tool. Once all leaves have been proven this way, one can easily build the proof of the initial termination property by applying lemmas from leaves to the root:

Lemma final: well_founded R.

Proof. apply (wf_R_if_wf_Ri wf_R1 wf_R2 ...). Qed.

\subsection{The running example}

We illustrate our method with a very simple TRS $R=R_{\text {ack }} \cup R_{\text {add }}$ (over a signature $\mathcal{F}$ ) where $R_{\text {ack }}$ computes the Ackerman function on Peano integers, and $R_{\text {add }}$ computes addition on binary integers. The digits are denoted as postfix operators (_) 0 and (_)1, whereas \# is the constant 0 seen as a number, shared between Peano integers and binary ones.

$$
R\left\{\begin{array}{l}
R_{\text {ack }}\left\{\begin{array}{l}
\operatorname{ack}(\#, y) \rightarrow s(y) \quad \operatorname{ack}(s(x), \#) \rightarrow \operatorname{ack}(x, s(\#)) \\
\operatorname{ack}(s(x), s(y)) \rightarrow \operatorname{ack}(x, \operatorname{ack}(s(x), y))
\end{array}\right. \\
R_{\text {add }} \begin{cases}(\#) 0 \rightarrow \# \quad x+\# \rightarrow x \\
(x) 0+(y) 0 \rightarrow(x+y) 0 & (x) 1+(y) 0 \rightarrow(x+y) 1 \\
(x) 0+(y) 1 \rightarrow(x+y) 1 & (x) 1+(y) 1 \rightarrow((x+y)+(\#) 1) 0\end{cases}
\end{array}\right.
$$

\subsection{Generation of the TRS definition}

For sake of clarity we will use CoQ notations that are different than in previous sections:

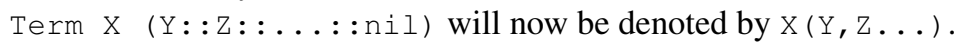

The generation of the $\Sigma$-algebra corresponding to a signature in the automated tool is straightforward. We show here the signature corresponding to the $\Sigma$-algebra of $\mathcal{F}$. Notice the module type constraint $<$ : Signature making CoQ check that definitions and properties of SIGMA_F comply with Signature as defined in Section 3.2.

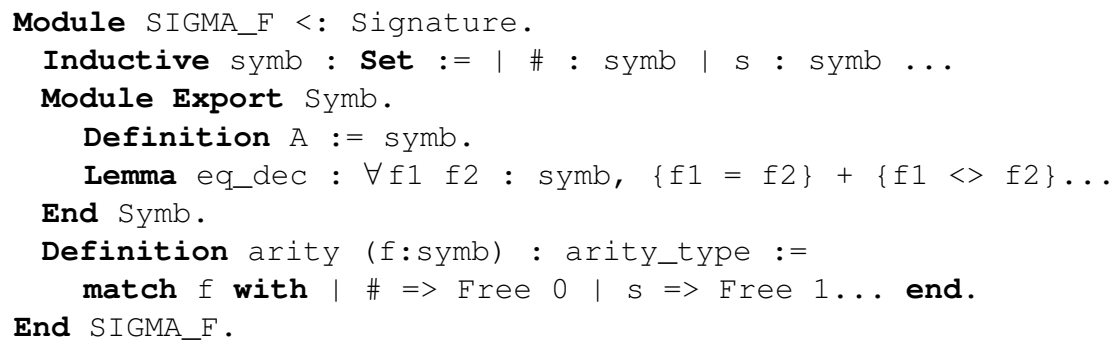

We define a module VARS for variables, apply functors building the term algebra and rewrite system on it, and then the rewriting system corresponding to $R$ :

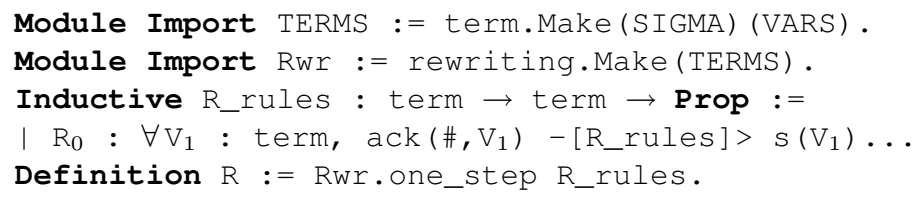

Notice that from now on notation $T-[R]>U$ denotes that $T$ rewrites to $U$ in the sense of section 2.1, i.e. there exists two subterms $t$ and $u$ at the same position in respectively $\mathrm{T}$ and $\mathrm{U}$, such that $\mathrm{R} u t$ (see the definition of one_step in section 3.2). 


\subsection{Criterion: Dependency Pairs}

The (unmarked) dependency pairs of $R$ generated by CiME are the following:

$$
\begin{aligned}
& \langle\operatorname{ack}(s(x), \#), \operatorname{ack}(x, s(\#))\rangle \\
& \langle\operatorname{ack}(s(x), s(y)), \operatorname{ack}(x, \operatorname{ack}(s(x), y))\rangle \quad\langle\operatorname{ack}(s(x), s(y)), \operatorname{ack}(s(x), y)\rangle \\
& \langle(x) 1+(y) 0, x+y\rangle \quad\langle(x) 0+(y) 1, x+y\rangle \quad\langle(x) 0+(y) 0, x+y\rangle \quad\langle(x) 0+(y) 0,(x+y) 0\rangle \\
& \langle(x) 1+(y) 1, x+y\rangle \quad\langle(x) 1+(y) 1,(x+y)+(\#) 1\rangle \quad\langle(x) 1+(y) 1,((x+y)+(\#) 1) 0\rangle
\end{aligned}
$$

An inductive relation representing the dependency chains [1] is built automatically. A step of this relation models the (finite) reductions by $R$ in the strict subterms of DP instances (e.g. $x_{0} \rightarrow^{\star} s\left(V_{0}\right), \ldots$ ) and one step of the relevant dependency pair. We illustrate this on $\langle\operatorname{ack}(s(x), \#), \operatorname{ack}(x, s(\#))\rangle$ with $\sigma=\left\{x \mapsto V_{0}\right\}$ :

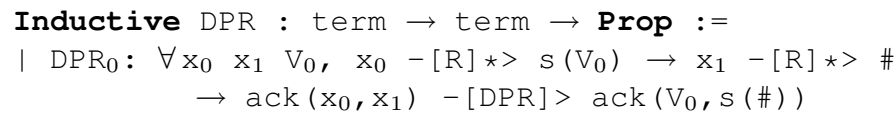

The main lemma on DPs fits in the general structure we explained on section 4.1:

Lemma wfR_if_wfDPR: well_founded DPR $\rightarrow$ well_founded R.

The proof follows a general scheme due to Hubert [21]. It involves several nested inductions instantiating the proof of the criterion in the particular setting of DPR and R.

Note that we can also prove this lemma in the case of an enhancement of DPs by Dershowitz [12] consisting in discarding DPs whose rhs is a subterm of the $1 \mathrm{hs}{ }^{8}$.

Marked symbols A refinement of the DP criterion consists in marking head symbols in lhs and rhs of dependency pairs in order to relax ordering constraints. We simply generate the symbol type with two versions of each symbol and adapt the definition of orderings. The proof strategy needs no change.

\subsection{Criterion: Dependency Pairs with graph}

Not all DPs can follow one another in a dependency chain: one may consider the graph of possible sequences of DPs (dependency graph). This graph is not computable, so one uses graphs containing it. We consider here Arts \& Giesl's simple approximation [1].

The graph criterion [1] takes benefit from working on the (approximated) graph. In its weak version, it consists in providing for each strongly connected component (SCC) an ordering pair that decreases strictly for all its nodes, and weakly for all rules. In its strong version, it considers cycles:

Theorem 1 (Arts and Giesl [1]). A TRS $\mathcal{R}$ is terminating iff for each cycle $\mathcal{P}$ in its dependency graph there is a reduction pair $\left(\succeq_{\mathcal{P}}, \succ_{\mathcal{P}}\right)$ such that: $(1) l \succeq_{\mathcal{P}} r$ for any $l \rightarrow r \in \mathcal{R}$, (2) $s \succeq_{\mathcal{P}} t$ for any $\langle s, t\rangle \in \mathcal{P}$, and (3) $s \succ_{\mathcal{P}} t$ for at least one pair in $\mathcal{P}$.

\footnotetext{
${ }^{8}$ Such DPs cannot occur in minimal chains. Thus they can be discarded.
} 
In practice, our tool uses a procedure due to Middledorp and Hirokawa [19] which splits recursively the graph into sub-components using different orders. The proof uses shallow embedding. One reason for this choice is that a generic theorem for a complex graph criterion is not easy to prove since it involves a substantial part of graph theory (e.g. the notion of cycle). Moreover, verifying the premises of such a theorem amounts to checking that all SCCs found by the prover are really SCCs and that they are terminating, but also to proving that it found all SCCs of the graph. That is tedious. On the contrary, using shallow embedding we use these facts implicitly by focusing on the termination proof of each component.

Weak version The first thing we generate is the definition of each component as computed by CiME. To illustrate the graph criterion on our example we may take the whole system $R$. CiME detects two components (sub 0 with some DPs of $R_{\text {add }}$, sub 1 with some DPs of $R_{\text {ack }}$ ): we generate the two corresponding sub-relations of DPR.

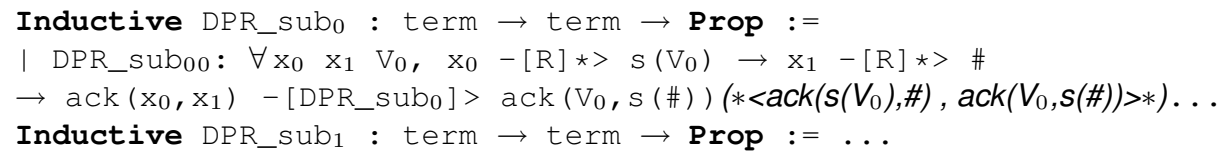

The following lemma states the criterion and fits the general structure in Section 4.1.

Lemma wf_DPR_if_wf_subo_sub 1 : well_founded DPR_subo $\rightarrow$ well_founded DPR_sub1 $\rightarrow$ well_founded DPR.

The proof of these lemmas uses the idea that if we collapse each SCC into one node, they form a DAG on which we can reason by cases on the edges in a depth-first fashion.

Strong version In addition, when the strong version of the criterion is used, the termination of each sub-component may itself be proven from the termination of smaller components, each one with a different ordering. Due to lack of space, we will not go into the details of this methodology.

It remains to conclude by providing well-suited ordering pairs.

\subsection{Orderings: Polynomial interpretations}

In our framework a polynomial interpretation is defined as a recursive function on terms. $\mathrm{C} i \mathrm{ME}$ outputs an interpretation for the SCC subo (other symbols are mapped to 0 ):

$$
[\#]=0 ;[0]\left(X_{0}\right)=X_{0}+1 ;[1](X 0)=X_{0}+1 ;[+](X 0, X 1)=X 1+X_{0} \text {; }
$$

From this interpretation we produce a measure: term $\rightarrow \mathrm{z}$ :

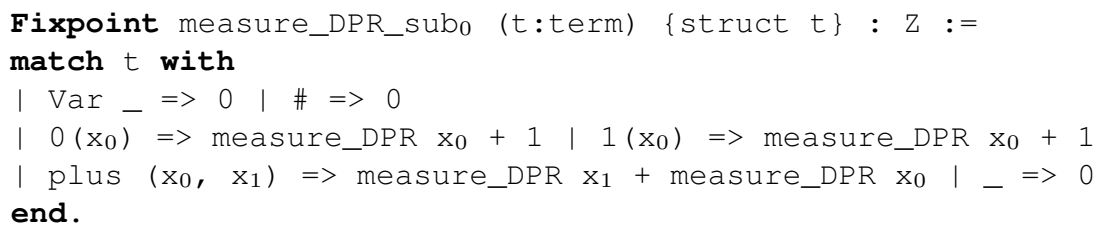


Notice that although our term definition is a deep embedding, the measure is defined as if we were in a shallow embedding. Indeed it is defined by a direct recursive function on terms and does not refer to polynomials, substitutions or variables $\left(x_{0}\right.$ above is a CoQ variable, it is not a rewriting variable which would be of the form Var $n$ ). This choice makes, once again, our proofs simpler to generate. In a deep embedding we would need a theory for polynomials, and a generic theorem stating that a polynomial on positive integers with positive factors is monotonic. But actually this property instantiated on measure_DPR_sub 0 above can be proven by a trivial induction on $t$. So again the effort of a deep embedding is not worth this effort. The following Lemma proves the well-foundedness of measure_DPR_subo:

Lemma Well_founded_DPR_sub 0 : well_founded DPR_sub .

which is equivalent to $\forall x, \operatorname{ACC}$ DPR $x$. This is proven firstly by induction on the value of (measure_DPR_subo $x$ ), then by cases on each DP of DPR_subo, finally by applying the induction hypothesis using the fact that each pair is decreasing by measure_DPR_subo. One concludes by polynomial comparison. It is well known that the comparison of non-linear polynomials on $\mathbb{N}$ is not decidable in general. We have a decision procedure for the particular kind of non linear polynomials CiME produces.

\subsection{Orderings: RPO}

The COCCINELLE library formalises RPO in a generic way, and proves it to be wellsuited for ordering pairs. RPO is defined using a precedence (a decidable strict ordering prec over symbols) and a status (multiset/lexicographic) for each symbol.

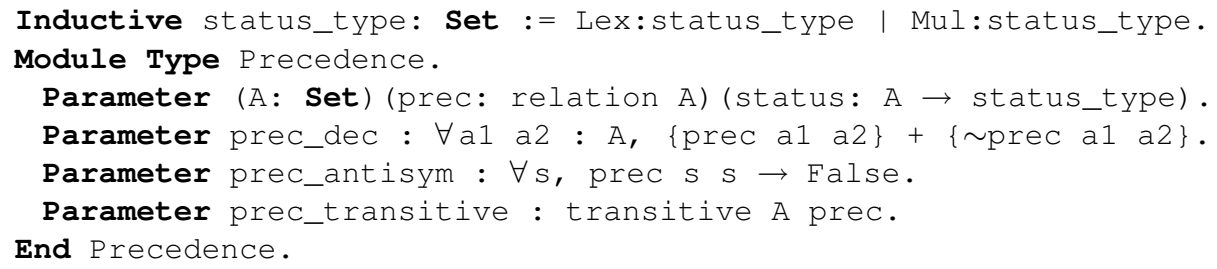

A module type for an RPO should be built from a term algebra and a precedence:

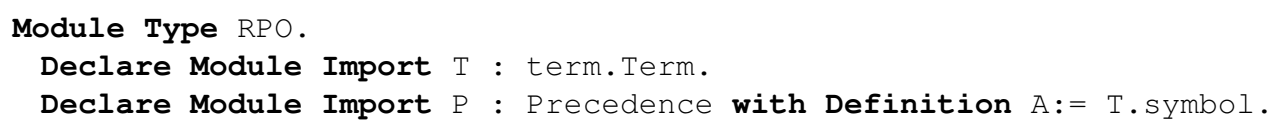

The library contains a functor rpo. Make building an RPO from two modules of type Term and Precedence. It also builds among other usual properties of RPO, the proof that if the precedence is well-founded, then so is the RPO. This part of the library is in a deep embedding style. Proofs of termination using RPOs are very easy to generate as it is sufficient to generate the precedence, the proof that it is well-founded and to apply the functor rpo. Make. It should be noticed that the fact that the generic RPO uses a strict precedence and a comparison from left to right in the lexicographic case is not a restriction in practice: a simple translation from terms to terms mapping equivalent symbols onto the same symbol, and performing the wanted permutation over the subterms under a given lexicographic symbol is both monotonic and stable. Hence 
the relation defined by comparing the translations of terms by the generic RPO still has the desired properties.

The generated definition of the RPO used for proving well-foundedness of $\mathrm{sub}_{1}$ is:

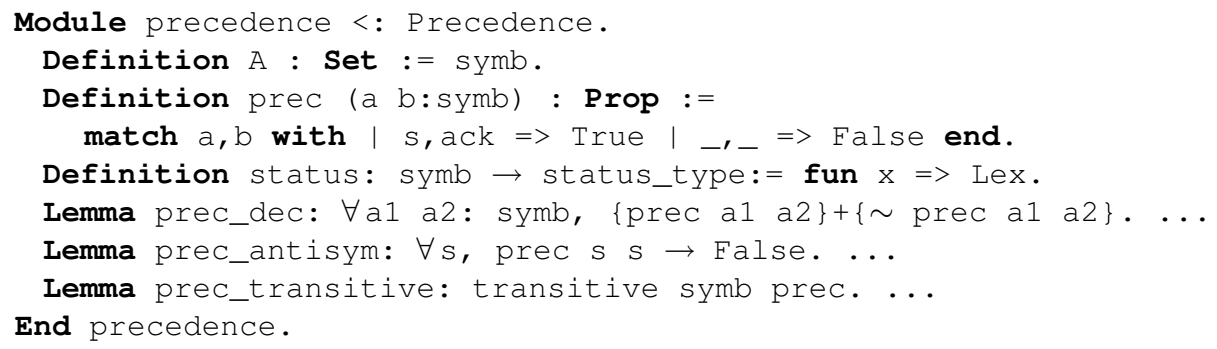

And as previously: Lemma Well_founded_DPR_sub 1 : well_founded DPR_sub .

Argument filtering systems The use of Dependency Pairs allows a wide choice of orderings by dropping the condition of strict monotonicity. Regarding path orderings, this can be achieved using argument filtering systems (AFS) [1]. We define AFSs as fixpoints and apply them at comparison time. This does not affect the (COQ) proof scheme.

\section{Results and benchmarks}

CiME 2.99 can be downloaded and tested from the A3PAT website ${ }^{9}$. Once the system is defined, we have to choose the termination criterion and the orderings. For instance, we may select DP with graphs refinement and both linear polynomials (bound 2) and RPO with AFSs, then ask CiME to check termination and generate the proof trace:

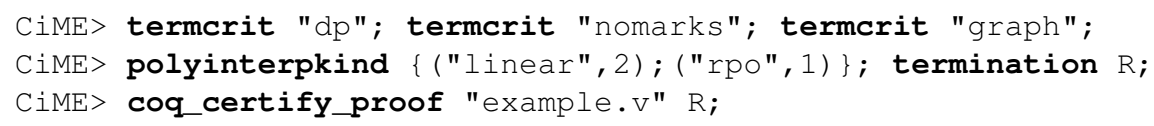

We used the Termination Problems Data Base ${ }^{10}$ V3.2 as challenge. Until now we have produced a COQ certificate for $100 \%$ of the 358 TRS that CiME proves terminating without using modular technique or $\mathrm{AC}$ termination ${ }^{11}$. We will now give some details on our experiments. We give below, depending on the use of graphs, the average and max. sizes of compiled COQ proofs, as well as the average compilation time (together with the number of problem solved) using marks on a $2 \mathrm{GHz}, 1 \mathrm{~GB}$ machine, running Linux. RPO + Pol. means that selected orderings for proof search are RPOs and polynomials.

\begin{tabular}{|c|rrrr|rrrr|}
\hline & \multicolumn{5}{|c|}{ with graph } & \multicolumn{4}{c|}{ without graph } \\
& s. av. & s. max & t. av & $(\mathrm{nb})$ & s. av. & s. max & t. av & ( nb ) \\
\hline RPO & $3.71 \mathrm{MB}$ & $9.1 \mathrm{MB}$ & $27.3 \mathrm{~s}$ & $(202)$ & $3.52 \mathrm{MB}$ & $4.77 \mathrm{MB}$ & $21.2 \mathrm{~s}$ & $(123)$ \\
\hline Linear Pol. & $0.76 \mathrm{MB}$ & $3.86 \mathrm{MB}$ & $13.6 \mathrm{~s}$ & $(231)$ & $0.51 \mathrm{MB}$ & $4.56 \mathrm{MB}$ & $8.1 \mathrm{~s}$ & $(175)$ \\
\hline Simple Pol. & $0.91 \mathrm{MB}$ & $10.91 \mathrm{MB}$ & $30.3 \mathrm{~s}$ & $(274)$ & & & & \\
\hline RPO + Pol. & $1.27 \mathrm{MB}$ & $11 \mathrm{MB}$ & $21.5 \mathrm{~s}$ & $(358)$ & $1.99 \mathrm{MB}$ & $7.39 \mathrm{MB}$ & $12.7 \mathrm{~s}$ & $(255)$ \\
\hline
\end{tabular}

\footnotetext{
${ }^{9}$ http://www3.ensiie.fr/ urbain/a3pat/pub/index.en.html

10 http: / / www. lri.fr/ marche/tpdb

${ }^{11}$ Not all the systems of the TPDB are terminating. Some are proven by the full termination engine of $\mathrm{CiME} 2.04$ using techniques for which $\mathrm{CiME} 2.99$ does not produce a certificate yet.
} 


\section{Related works and conclusion}

There are several works to be mentioned w.r.t. the communication between automated provers and COQ. Amongst them, the theorem-prover ZÉNON [14], based on tableaux, produces COQ proof terms as certificates. ELAN enjoys techniques to produce COQ certificates for rewriting [26]. Bezem describes an approach regarding resolution [3]. However, these systems do not tackle the problem of termination proofs.

To our knowledge the only other approach to generate termination certificates for rewriting systems relies on the CoLoR/Rainbow libraries [4]. In this approach, term algebras and TRSs are handled via an embedding even deeper than in COCCINELLE, since a TRS is given by a set of pairs of terms. Rainbow is a relatively efficient tool thanks to orderings built with matrix interpretations (which we don't handle yet). But it does not handle the following techniques: enhanced or marked dependency pairs, complex graphs, RPO with AFS. We think that adding these techniques to CoLoR/Rainbow will be hard, due to the pure deep embedding approach. There are currently 167 out of 864 termination problems in TPDB (v3.2) proven by TPA [22] and certified by CoLoR/Rainbow using polynomial interpretations and the webpage mentions 237 problems certified using matrix interpretations.

We presented a methodology to make automated termination tools generate traces in a proof assistant format. The approach is validated by a prototype generating COQ traces. The performances of the prototype on the examples of the TPDB database are promising. Our approach is easy to extend, in particular because extensions may be done in deep or shallow embedding.

To apply this methodology on different tools and targeted proof assistants, one needs a termination trace language. An ongoing work in the A3PAT group is to define a more general language that can even tackle proofs of various rewriting properties such as termination, confluence (which needs termination), equational proofs [7], etc. We think that a good candidate could be based on the tree structure we explained on Section 4.1.

One particularly interesting follow-up of this work is the possibility to plug automated termination tools as external termination tactics for proof assistants. Indeed termination is a key property of many algorithms to be proven in proof assistants. Moreover, in type theory based proof assistants like CoQ, one cannot define a function without simultaneously proving its termination. This would allow to define functions whose termination is not obvious without the great proof effort it currently needs.

\section{References}

1. T. Arts and J. Giesl. Termination of term rewriting using dependency pairs. Theoretical Computer Science, 236:133-178, 2000.

2. F. Baader and T. Nipkow. Term Rewriting and All That. Cambridge University Press, 1998.

3. M. Bezem, D. Hendriks, and H. de Nivelle. Automated proof construction in type theory using resolution. J. Autom. Reasoning, 29(3-4):253-275, 2002.

4. F. Blanqui, S. Coupet-Grimal, W. Delobel, S. Hinderer, and A. Koprowski. Color, a coq library on rewriting and termination. In Geser and Sondergaard [16]. 
5. E. Contejean. A certified AC matching algorithm. In V. van Oostrom, editor, 15th International Conference on Rewriting Techniques and Applications, volume 3091 of Lecture Notes in Computer Science, pages 70-84, Aachen, Germany, June 2004. Springer-Verlag.

6. E. Contejean. Coccinelle, 2005. http://www.lri.fr/ contejea/Coccinelle/ coccinelle.html.

7. E. Contejean and P. Corbineau. Reflecting proofs in first-order logic with equality. In 20th International Conference on Automated Deduction (CADE-20), number 3632 in Lecture Notes in Artificial Intelligence, pages 7-22, Tallinn, Estonia, July 2005. Springer-Verlag.

8. E. Contejean, C. Marché, B. Monate, and X. Urbain. Proving termination of rewriting with Cime. In Rubio [28], pages 71-73. http: / / cime. lri.fr.

9. T. Coquand and C. Paulin-Mohring. Inductively defined types. In P. Martin-Löf and G. Mints, editors, Proceedings of Colog'88, volume 417 of Lecture Notes in Computer Science. Springer-Verlag, 1990.

10. N. Dershowitz. Orderings for term rewriting systems. Theoretical Computer Science, 17(3):279-301, Mar. 1982.

11. N. Dershowitz. Termination of rewriting. Journal of Symbolic Computation, 3(1):69-115, Feb. 1987.

12. N. Dershowitz. Termination Dependencies. In Rubio [28]. Technical Report DSIC II/15/03, Univ. Politécnica de Valencia, Spain.

13. N. Dershowitz and J.-P. Jouannaud. Rewrite systems. In J. van Leeuwen, editor, Handbook of Theoretical Computer Science, volume B, pages 243-320. North-Holland, 1990.

14. D. Doligez. Zenon. http://focal.inria.fr/zenon/.

15. J. Endrullis. Jambox. http://joerg.endrullis.de/index.html.

16. A. Geser and H. Sondergaard, editors. Extended Abstracts of the 8th International Workshop on Termination, WST'06, Aug. 2006.

17. J. Giesl, P. Schneider-Kamp, and R. Thiemann. Aprove 1.2: Automatic termination proofs in the dependency pair framework. In U. Furbach and N. Shankar, editors, Third International Joint Conference on Automated Reasoning, volume 4130 of Lecture Notes in Computer Science, Seattle, USA, Aug. 2006. Springer-Verlag.

18. J. Giesl, R. Thiemann, P. Schneider-Kamp, and S. Falke. Mechanizing and Improving Dependency Pairs. Journal of Automated Reasoning, 37(3):155-203, 2006.

19. N. Hirokawa and A. Middeldorp. Automating the dependency pair method. In F. Baader, editor, 19th International Conference on Automated Deduction (CADE-19), volume 2741 of Lecture Notes in Computer Science, pages 32-46, Miami Beach, FL, USA, July 2003. Springer-Verlag.

20. N. Hirokawa and A. Middeldorp. Tyrolean termination tool. In J. Giesl, editor, 16th International Conference on Rewriting Techniques and Applications (RTA'05), volume 3467 of Lecture Notes in Computer Science, pages 175-184, Nara, Japan, Apr. 2005. SpringerVerlag.

21. T. Hubert. Certification des preuves de terminaison en Coq. Rapport de DEA, Université Paris 7, Sept. 2004. In French.

22. A. Koprowski. TPA. http://www.win.tue.nl/tpa.

23. D. S. Lankford. On proving term rewriting systems are Noetherian. Technical Report MTP3, Mathematics Department, Louisiana Tech. Univ., 1979. Available at http://perso. ens-lyon.fr/pierre. lescanne/not_accessible.html.

24. B. N. m. Terminating general recursion. BIT Numerical Mathematics.

25. C. Marché and H. Zantema. The termination competition 2006. In Geser and Sondergaard [16]. http://www.lri.fr/ marche/termination-competition/.

26. Q. H. Nguyen, C. Kirchner, and H. Kirchner. External rewriting for skeptical proof assistants. J. Autom. Reasoning, 29(3-4):309-336, 2002. 
27. T. Nipkow, L. C. Paulson, and M. Wenzel. Isabelle/HOL - A Proof Assistant for HigherOrder Logic, volume 2283 of Lecture Notes in Computer Science. Springer-Verlag, 2002.

28. A. Rubio, editor. Extended Abstracts of the 6th International Workshop on Termination, WST'03, June 2003. Technical Report DSIC II/15/03, Univ. Politécnica de Valencia, Spain.

29. The Coq Development Team. The Coq Proof Assistant Documentation - Version V8.1, Feb. 2007. http://coq.inria.fr.

30. X. Urbain. Modular and incremental automated termination proofs. Journal of Automated Reasoning, 32:315-355, 2004. 